\title{
EIT and TRACE responses to flare plasma
}

\author{
D. Tripathi ${ }^{1}$, G. Del Zanna ${ }^{2}$, H. E. Mason ${ }^{1}$, and C. Chifor ${ }^{1}$ \\ 1 Department of Applied Maths and Theoretical Physics, University of Cambridge, Cambridge CB3 OWA, UK \\ e-mail: [D.Tripathi;H.E.Mason;C.Chifor]@damtp.cam.ac.uk \\ 2 University College London, MSSL, Holmbury St. Mary Dorking Surrey RH5 6NT, UK \\ e-mail: gd232@mssl.ucl.ac.uk
}

Received 29 September 2006 / Accepted 2 November 2006

\section{ABSTRACT}

\begin{abstract}
Aims. To understand the contribution of active region and flare plasmas to the $\lambda 195$ channels of SOHO/EIT (Extreme-ultraviolet Imaging Telescope) and TRACE (Transition Region and Coronal Explorer).

Methods. We have analysed an M8 flare simultaneously observed by the Coronal Diagnostic Spectrometer (CDS), EIT, TRACE and RHESSI. We obtained synthetic spectra for the flaring region and an outer region using the differential emission measures (DEM) of emitting plasma based on CDS and RHESSI observations and the CHIANTI atomic database. We then predicted the EIT and TRACE count rates.

Results. For the flaring region, both EIT and TRACE images taken through the $\lambda 195$ filter are dominated by Fe XXIV (formed at about $20 \mathrm{MK}$ ). However, in the outer region, the emission was primarily due to the Fe XII, with substantial contributions from other lines. The average count rate for the outer region was within $25 \%$ the observed value for EIT, while for TRACE it was a factor of two higher. For the flare region, the predicted count rate was a factor of two (in case of EIT) and a factor of three (in case of TRACE) higher than the actual count rate.

Conclusions. During a solar flare, both TRACE and EIT $\lambda 195$ channels are found to be dominated by Fe XXIV emission. Reasonable agreement between predictions and observations is found, however some discrepancies need to be further investigated.
\end{abstract}

Key words. Sun: corona - Sun: activity - Sun: flares - techniques: spectroscopic

\section{Introduction}

The Extreme-ultraviolet Imaging Telescopes (EIT; Delaboudinière et al. 1995) and the Coronal Diagnostic Spectrometer (CDS; Harrison et al. 1995) aboard the Solar and Heliospheric Observatory (SOHO) and the Transition Region and Coronal Explorer (TRACE; Handy et al. 1998) have provided a wealth of information about the different, highly dynamic layers of solar atmosphere. In order to understand the physics, it is mandatory to understand the properties of plasma which is being observed. Multi-wavelength observations from different instruments together with the CHIANTI atomic database (Dere et al. 1997; v.5; Landi et al. 2006) are routinely used to study a variety of different solar features.

The EIT provides full disk observations of the Sun in four spectral bands, one of which is centered around 2195 . In normal quiet Sun conditions, this band is dominated by Fe XII emission, while in coronal hole plumes it is dominated by Fe VIII (Del Zanna et al. 2003). It has been suggested (e.g., McKenzie 2000) that this channel may be contaminated by hightemperature Fe XXIV (20 MK) emission. However this has not been throughly studied or definitively proven.

The TRACE also provides observations of the solar corona in three coronal band, one of which is also centered around $\lambda 195$. It has been proposed that the TRACE $\lambda 195$ channel has a high-temperature response due to Fe XXIV during flares. There is substantial evidence in the literature where Fe XXIV emission is likely to be dominant, by comparisons of TRACE $\lambda 195 \mathrm{im}-$ ages with Yohkoh/SXT (e.g. Warren et al. 1999), RHESSI (e.g.
Gallagher et al. 2002; Berlicki et al. 2004; Phillips et al. 2005), or spectroscopic data (e.g. Del Zanna et al. 2006). However, no direct quantitative estimates of the importance of Fe XXIV emission (for either EIT and TRACE) have previously been made. To our knowledge, only Warren et al. (1999) have attempted to simulate the TRACE $\lambda 195$ count rates of a flare in an indirect way, by using SXT filter ratios. These authors obtained predicted count rates about 3 times higher than those observed.

Both EIT and TRACE have provided many images of flares, and it is therefore important to establish the Fe XXIV contribution to this band. This is not a trivial task, since simultaneous multiwavelength observations of flares are a rare event. In this paper, we have combined simultaneous observations of an M8 flare obtained with CDS, RHESSI, EIT and TRACE $\lambda 195$ images. We have derived the differential emission measure (DEM) from CDS and RHESSI data for a flaring region and an outer region and then performed forward modelling with the CHIANTI atomic database to predict the EIT and TRACE count rates.

\section{Observations and data reduction}

We performed a search through the entire CDS NIS (Normal Incidence Spectrometer) database to find suitable flare events. Out of the many flare datasets recorded by CDS, only an M8class flare on 28-Oct.-2003 at around 10:48 UT was simultaneously detected by EIT, TRACE $\lambda 195$ and RHESSI (Lin et al. 2002). This flare occurred in the active region AR0486 located at S18 E18. Figure 1 displays the GOES-12 plot for this flare. The 


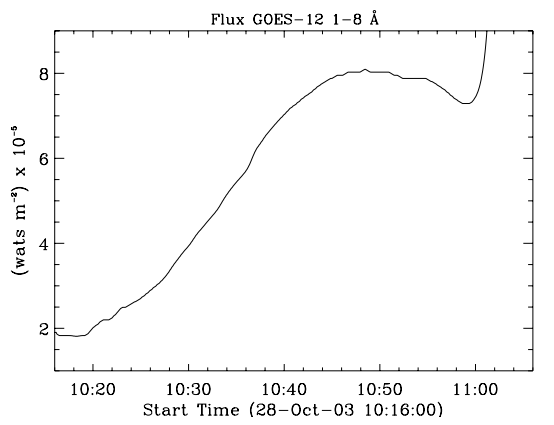

Fig. 1. The GOES-12 plot for the flare in the 1.0-8.0 Å channel.

flare started around 10:20 UT with a peak at around 10:45 UT and ended at around 11:00 UT.

Figure 2 displays the images recorded by CDS in Fe XIX line, EIT and TRACE $\lambda 195$ with overlayed Fe XIX and RHESSI intensity contours. The RHESSI image was obtained at 10:33 UT by integrating the counts for $20 \mathrm{~s}$. Note that the flare at location $(-280,-380)$ arcsec is of relevance in this paper as this was observed simultaneously by all instruments. The CDS raster scan started at 09:37:40 UT (Solar $X=-90$, see Fig. 2) and it observed the peak flare emission (Solar $X=-250$ :-300) between 10:33 and 10:47 UT i.e., around the peak of the flare.

We have examined the sequence of TRACE (cadence: $\approx 1 \mathrm{~min}$ ) and EIT (cadence: $12 \mathrm{~min}$ ) images and found that the flare region varied in intensity by less than $20 \%$ during the CDS scan, as expected when considering the GOES light curve.

The standard routines provided in solar software (SSW) were used for processing the EIT and TRACE images. The CDS data processing was performed using routines and calibration described in Del Zanna et al. (2001). In order to locate exactly the same region we coaligned the images obtained by CDS, EIT and TRACE. The TRACE image taken at 10:47 UT was first coaligned with the EIT images and then the CDS intensity contours were overplotted. As can be seen from Fig. 2, Fe XIX and RHESSI intensity contours match extremely well the peak emission in the EIT and TRACE images.

\section{Forward and inverse modelling}

The intensity of an optically thin emission line can be written as

$I=A(Z) \int_{T_{\mathrm{e}}} G\left(T_{\mathrm{e}}, N_{\mathrm{e}}\right) \operatorname{DEM}\left(T_{\mathrm{e}}\right) \mathrm{d} T_{\mathrm{e}}$

where, $A(Z)$ is the elemental abundance, $T_{\mathrm{e}}$ is the electron temperature, and $N_{\mathrm{e}}$ is the electron density, $G\left(T_{\mathrm{e}}, N_{\mathrm{e}}\right)$ is the contribution function which contains all the relevant atomic physics coefficients. $\operatorname{DEM}\left(T_{\mathrm{e}}\right)$ is defined as $\operatorname{DEM}\left(T_{\mathrm{e}}\right)=N_{\mathrm{e}}^{2}\left(\mathrm{~d} h / \mathrm{d} T_{\mathrm{e}}\right)$, where $\mathrm{d} h$ is an element of column height along the line-of-sight.

To calculate the $\operatorname{DEM}\left(T_{\mathrm{e}}\right)$ the maximum entropy method described by Monsignori Fossi \& Landini (1991) and implemented by Del Zanna (1999) was used. In this method the DEM is assumed to be a series of spline mesh points, covering the temperature range for which the observational constraints are present. We measured the DEM of the flare region and an outer region shown in Fig. 3. Tables 1 and 2 represent the lines in the flare and outer regions respectively which where used for DEM analysis. As can be seen from the table, we have been able to predict the intensities to within 30\% using the derived DEM. The theoretical intensities were calculated by interfacing DEMs within CHIANTI using a constant electron density of $10^{11} \mathrm{~cm}^{-3}$ for the
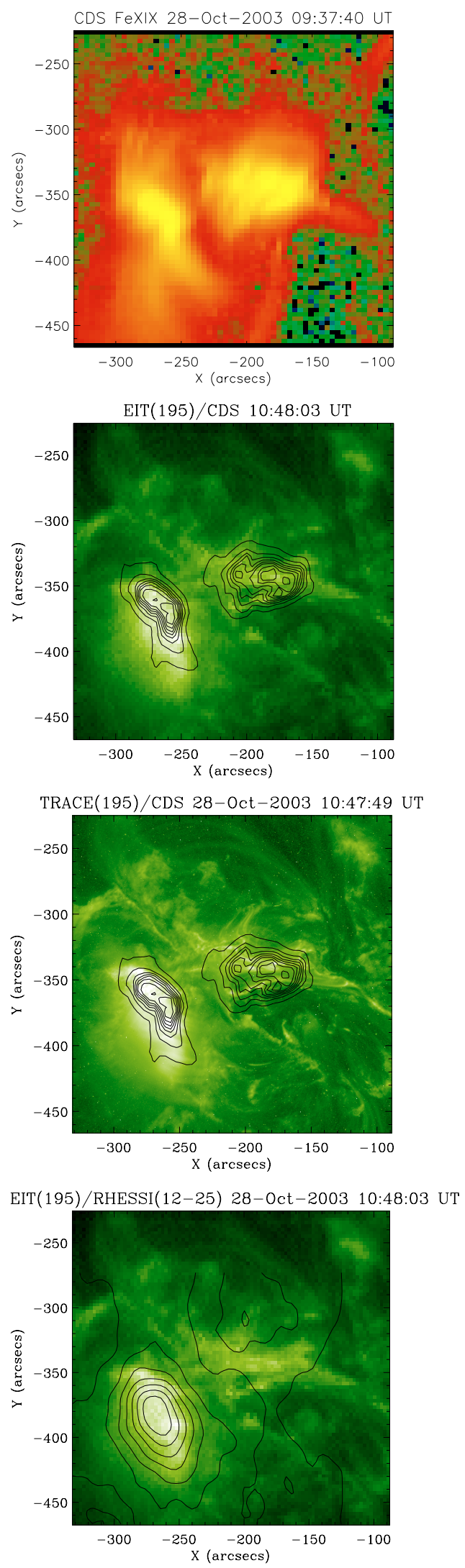

Fig. 2. A CDS image (top panel) in the Fe XIX ( $2592.2, \log T=7.0)$ line. The time 09:37:40 UT in the title is the start time of the raster. Images taken by EIT (second panel) and TRACE (third panel) respectively at $\lambda 195$ at 10:48:03 UT. The intensity contours from the CDS image (in the top panel) obtained for Fe XIX line are over plotted. The EIT images are overlayed with RHESSI contours in the $12-25 \mathrm{keV}$ channel (bottom panel). 


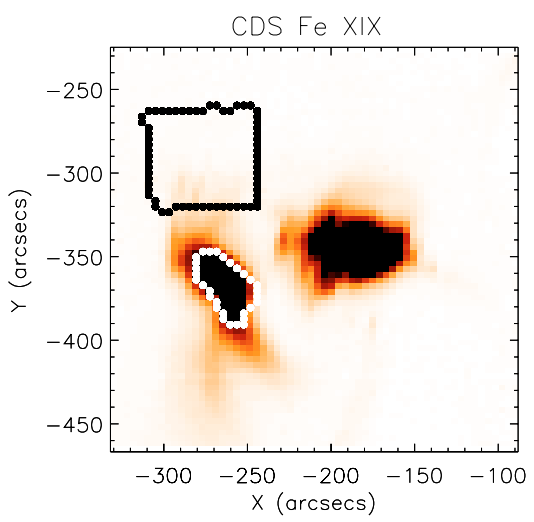

Fig. 3. CDS negative intensity image in Fe XIX showing the two regions selected for further analysis. White contour outlines the flare region and the black outlines the outer region.

Table 1. Intensities in the flare region from the CDS NIS. Note that the intensity of Fe XXV is deduced from RHESSI observations. In the table from left to right: the ion, the observed wavelength, the maximum formation temperature, the observed and predicted intensities (phot $\mathrm{cm}^{-2} \mathrm{sr}^{-1} \mathrm{~s}^{-1}$ ) and the ratio of the latter two.

\begin{tabular}{lccrrr}
\hline \hline Ion & $\begin{array}{c}\lambda_{\text {obs }} \\
(\AA)\end{array}$ & $\begin{array}{c}\log T_{\max } \\
(K)\end{array}$ & $I_{\text {obs }}$ & $I_{\text {dem }}$ & Ratio \\
\hline Ne IV & 543.9 & 5.3 & 381.4 & 301.8 & 0.79 \\
O IV & 553.3 & 5.3 & 1023.1 & 1029.8 & 1.01 \\
O V & 629.7 & 5.4 & 6507.1 & 5254.3 & 0.81 \\
Fe XI & 369.2 & 6.1 & 159.8 & 91.5 & 0.57 \\
Fe XII & 364.5 & 6.1 & 813.9 & 607.9 & 0.75 \\
Fe XIII & 348.2 & 6.2 & 266.9 & 255.9 & 0.96 \\
Fe XIV & 334.2 & 6.2 & 1199.4 & 1245.9 & 1.04 \\
Fe XVI & 335.3 & 6.3 & 27134.7 & 25142.0 & 0.93 \\
Mg X & 624.9 & 6.0 & 3339.3 & 4230.2 & 1.27 \\
Fe XIX & 592.2 & 7.0 & 2368.7 & 2354.8 & 0.99 \\
Fe XXV & 1.833 & 8.1 & 418305.1 & 286297.1 & 0.68 \\
\hline
\end{tabular}

flare region and $10^{9} \mathrm{~cm}^{-3}$ for the outer region, the ionization fraction of Arnaud \& Rothenflug (1985) and photospheric abundances. However, we note that the choice of ionization fraction and abundances has a negligible effect on the results.

In order to obtain the spectra, theoretically calculated line intensities were convolved with the effective areas of EIT $\lambda 195$ clear and TRACE $\lambda 195$ AO filters. The final spectra are shown in Fig. 4. The difference in the spectral resolution evident from Fig. 4 is due to the different sampling in the (measured) instrument effective areas. Note that in calculating the synthetic spectra, the continuum was included. From Fig. 4 it can be seen that the spectrum of the flare region (top panels) shows a strong peak at $\lambda 192$ due to Fe XXIV (20 MK) emission for both EIT and TRACE. However the spectra of the outer region (bottom panels) are dominated in TRACE by Fe XII lines. The differences between TRACE and EIT responses are significant. The EIT emission has a substantial contribution from other lines such as Fe XII $\lambda 187$, Fe XI $\lambda 188.2$ and 188.3, S XI $\lambda 188.7$ and $\lambda 191.25$, Fe XII $\lambda 192.4 \& \lambda 193.5$ and Fe XIII $\lambda 202.1$.

Finally the average count rates obtained from the synthetic spectra were compared with actual count rates obtained using the processed images. Table 3 provides the average count rates for EIT and TRACE corresponding to the flare and outer regions. As can be seen from Table 3 we could predict the average count rate for EIT within $25 \%$ for the outer region. However, the predicted count rate for the flare region is a factor of two higher. In the case
Table 2. Same as Table 1 but for the outer region.

\begin{tabular}{lccrrr}
\hline \hline Ion & $\begin{array}{c}\lambda_{\text {obs }} \\
(\AA)\end{array}$ & $\begin{array}{c}\log T_{\max } \\
(K)\end{array}$ & $I_{\text {obs }}$ & $I_{\text {dem }}$ & Ratio \\
\hline O IV & 554.1 & 5.3 & 102.0 & 125.2 & 1.23 \\
O V & 629.7 & 5.4 & 1067.8 & 346.0 & 0.32 \\
Fe XI & 369.2 & 6.1 & 38.5 & 29.5 & 0.77 \\
Mg IX & 368.1 & 6.0 & 441.4 & 430.1 & 0.97 \\
Fe XII & 364.5 & 6.1 & 247.8 & 304.9 & 1.23 \\
Fe XIV & 334.2 & 6.2 & 401.7 & 717.7 & 1.79 \\
Fe XVI & 335.3 & 6.3 & 4022.7 & 3404.5 & 0.85 \\
\hline
\end{tabular}

of TRACE, the predicted count rate is a factor of two higher in the outer region and a factor of 3 higher in the flare region.

\section{Discussion}

Using a similar forward modelling technique for the quiet Sun, Brooks \& Warren (2006) were able to predict the count rates within $25 \%$ for both EIT and TRACE $\lambda 195$ channel using data recorded in 1998. Their result matches well with the one obtained here for the outer region in the case of EIT but shows significant differences for the TRACE count rates. This could be due to the fact that the TRACE detector efficiency has decreased with time (as in the case of EIT). The current EIT analysis software applies a correction factor of about 7 for the dataset considered here, to take into account decreased sensitivity. This suggests that the TRACE calibration might need to be revisited. Considering that most emission is due to Fe XII, we can rule out any problems with the atomic data (Del Zanna \& Mason 2005). However, if the analysis presented by Warren et al. (1999) is correct, it suggests that calibration problems for TRACE were already present as early as July 1999 .

The question also arises that, why is there such a big difference in the observed and predicted count rates in the flare region? There could be at least two possible reasons. The first is that our DEM is over estimated. This could be due to the lack of constraint between the CDS Fe XIX $\lambda 592$ (8 MK) and the RHESSI Fe XXV $\lambda 1.8$ emission, or to an instrument calibration problem.

The second is that there remains some uncertainties in Fe XXIV emissivities. We note that strong discrepancies (factors 2-5) are common in other cooler Li-like ions (Del Zanna et al. 2002). This issue needs to be further explored, if EIT, TRACE (and soon STEREO, SDO) $\lambda 195$ images of flares are to be reliably used for plasma diagnostics.

Despite some differences in the count rates for the flare region for EIT and TRACE and in outer region for TRACE, we have clearly demonstrated that the EIT and TRACE $\lambda 195$ channels are dominated by Fe XXIV $\lambda 192$ emission during strong flares.

Future instruments, such as the Extreme-ultraviolet Imaging Spectrometer (EIS) aboard Hinode (Solar-B), will provide a better temperature coverage for the coronal component of active regions and flares. This will better constrain the predicted spectrum, enabling us to use CHIANTI to forward model the observational signal for various future missions.

Acknowledgements. We acknowledge the support of PPARC. We thank the SOHO/CDS, SOHO/EIT, TRACE, GOES and RHESSI team for providing the data. SOHO is an international collaboration between ESA and NASA. CHIANTI is a collaborative project involving the NRL (USA), RAL (UK), and the following Universities: College London (UK), Cambridge (UK), George Mason (USA), and Florence (Italy). GDZ warmly thanks DAMTP for the 

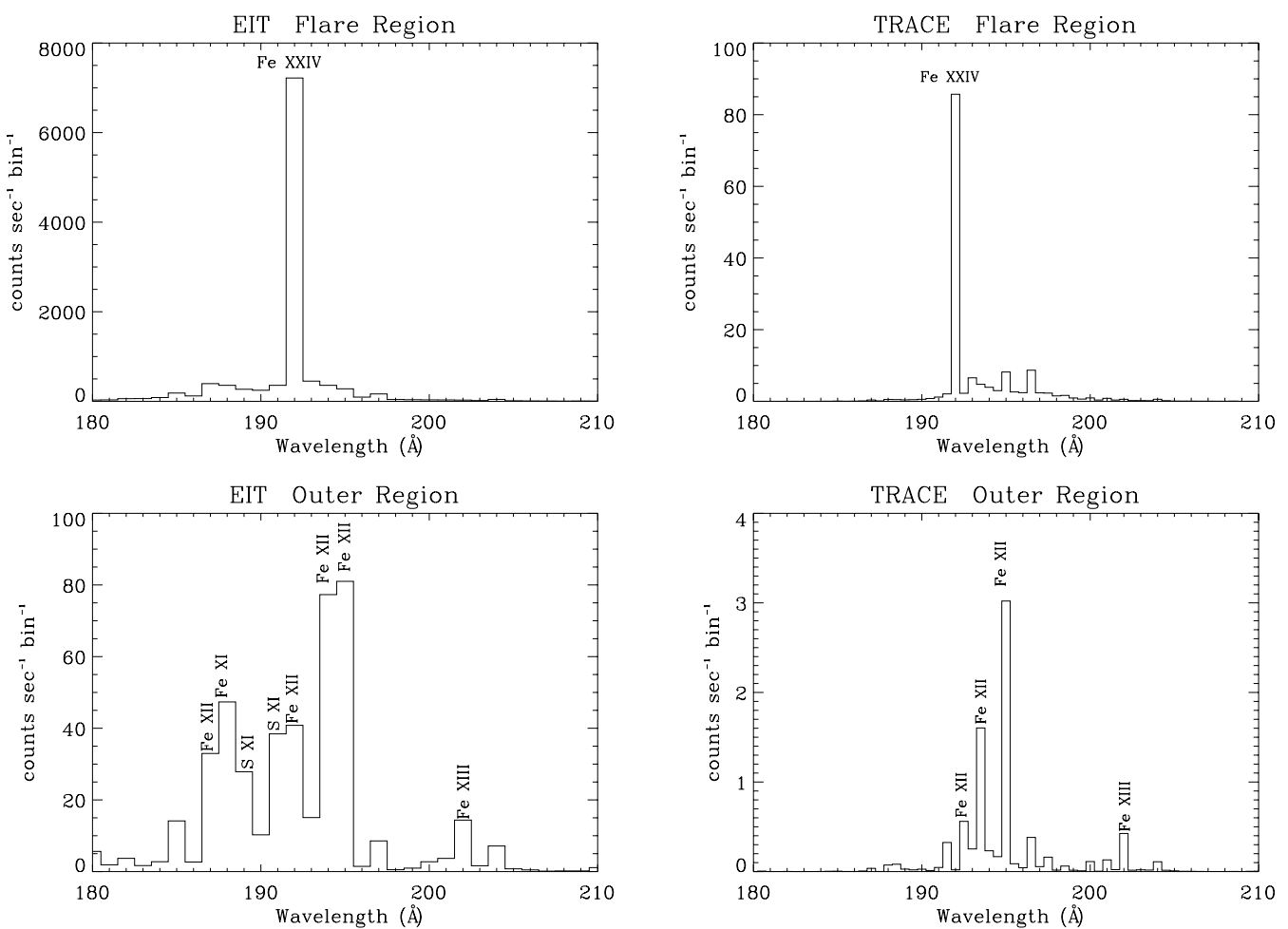

Fig. 4. CHIANTI synthetic spectra for the EIT (left hand side) and TRACE (right hand side) $\lambda 195$ channels in flare region (top panels) during a M8-class flare and outer region (bottom panels).

Table 3. Observed and predicted average count rates (DN s${ }^{-1}$ pixel $\left.^{-1}\right)$ for the regions marked in Fig. 3.

\begin{tabular}{ccccc}
\hline \hline & \multicolumn{2}{c}{ Flare region } & \multicolumn{2}{c}{ Outer region } \\
& Observed & Predicted & Observed & Predicted \\
\hline EIT & 6375.9 & 11035.66 & 572.5 & 447.37 \\
TRACE & 45.6 & 149.71 & 4.2 & 8.25 \\
\hline
\end{tabular}

hospitality during this collaboration. We thank Karel Schrijver for his help in understanding different filter combinations in TRACE.

\section{References}

Arnaud, M., \& Rothenflug, R. 1985, A\&AS, 60, 425 Berlicki, A., Schmieder, B., Vilmer, N., Aulanier, G., \& Del Zanna, G. 2004, A\&A, 423, 1119

Brooks, D. H., \& Warren, H. P. 2006, ApJS, 164, 202
Delaboudinière, J.-P., Artzner, G. E., Brunaud, J., et al. 1995, Sol. Phys., 162, 291

Del Zanna, G. 1999, Ph.D. Thesis, Univ. of Central Lancashire

Del Zanna, G., \& Mason, H. E. 2005, A\&A, 433, 731

Del Zanna, G., Bromage, B. J. I., Landi, E., \& Landini, M. 2001, A\&A, 379, 708

Del Zanna, G. M., Landini, \& Mason, H. E. 2002, A\&A, 385, 968

Del Zanna, G., Bromage, B. J. I., \& Mason, H. E. 2003, A\&A, 398, 743

Del Zanna, G., Berlicki, A., Mason, H. E., \& Schmieder, B. 2006, Sol. Phys., 234,95

Dere, K. P., Landi, E., Mason, H. E., Monsignori Fossi, B. C., \& Young, P. R. 1997, A\&AS, 125, 149

Gallagher, P. T., Dennis, B. R., Krucker, S., Schwartz, R. A., \& Tolbert, A. K. 2002, Sol. Phys., 210, 341

Handy, B. N., Acton, L. W., Kankelborg, C. C., et al. 1999, Sol. Phys., 187, 229

Harrison, R. A., Sawyer, E. C., Carter, M. K., et al. 1995, Sol. Phys., 162, 233

Landi, E., Del Zanna, G., Young, P. R., et al. 2006, 162, 261

Lin, R. P., Dennis, B. R., Hurford, G. J., et al. 2002, Sol. Phys., 210, 3

McKenzie, D. E. 2000, Sol. Phys., 195, 381

Monsignori Fossi, B. C., \& Landini, M. 1991, AdSpR, 11, 281

Phillips, K. J. H., Chifor, C., Dennis, B. R. 2006, ApJ, 647, 1480

Warren, H. P., Bookbinder, J. A., Forbes, T. G., et al. 1999, ApJ, 527, L121 